\title{
Lattice pulling effect and strain relaxation in axial (In,Ga)N/GaN nanowire heterostructures grown on GaN-buffered Si(111) substrate
}

\author{
X. Kong , S. Albert , A. Bengoechea-Encabo , M. A. Sanchez-Garcia , E. Calleja , and A. Trampert
}

Keywords axial (In,Ga)N/GaN nanowire, lattice pulling effect, plastic strain relaxation, transmission electron microscopy

Transmission electron microscopy and spatially resolved electron energy-loss spectroscopy have been applied to investigate the indium distribution and the interface morphology in axial (In,Ga)N/GaN nanowire heterostructures. The ordered axial $(\mathrm{In}, \mathrm{Ga}) \mathrm{N} / \mathrm{GaN}$ nanowire heterostructures with an indium concentration up to $80 \%$ are grown by molecular beam epitaxy on GaN-buffered $\mathrm{Si}(111)$ substrates. We observed a pronounced lattice pulling effect in all the nanowire samples given in a broad transition region at the interface. The lattice pulling effect becomes smaller and the (In, Ga)N/GaN interface width is reduced as the indium concentration is increased in the $(\mathrm{In}, \mathrm{Ga}) \mathrm{N}$ section. The result can be interpreted in terms of the increased plastic strain relaxation via the generation of the misfit dislocations at the interface.
1 Introduction The fabrication of three-dimensional axial $(\mathrm{In}, \mathrm{Ga}) \mathrm{N} / \mathrm{GaN}$ nanowire $(\mathrm{NW})$ heterostructures is of interest due to their potential as basic building blocks in future optoelectronic devices such as light emitting diodes $[1,2]$. However, there is a large lattice mismatch between $\mathrm{InN}$ and $\mathrm{GaN}$, which is on the order of $11 \%$ and will lead to internal elastic strain when ( $\mathrm{In}, \mathrm{Ga}) \mathrm{N}$ is epitaxially deposited on a $\mathrm{GaN}$ substrate. The elastic strain can produce a composition variation in ( $\mathrm{In}, \mathrm{Ga}) \mathrm{N}$ layers on $\mathrm{GaN}$, which is the so-called "lattice pulling" effect. In this effect, the lattice mismatch strain between $\mathrm{GaN}$ substrate and $(\mathrm{In}, \mathrm{Ga}) \mathrm{N}$ epilayer impedes the incorporation of indium, thus "pulling" the compositions of the growing $(\mathrm{In}, \mathrm{Ga}) \mathrm{N}$ layer towards that of the substrate, i.e., towards low indium concentration and thus towards lattice matching conditions. The lattice pulling effect has been observed in $(\mathrm{In}, \mathrm{Ga}) \mathrm{N}$ planar thin films $[3,4]$. Recently, we have also detected this effect in axial (In, Ga)N/GaN NW heterostructures [5]. However, in this three-dimensional NW geometry, a detailed study to which extent the lattice pulling effect can be influenced by the associated interface morpholgoy and the strain state is still absent.
Although progress has been made in measuring the local composition within individual NWs, it is still challenging to properly interpret the composition information mostly due to the strong coupling between the shape, strain and composition. We recently have demonstrated the potential of the low-loss electron energy-loss spectroscopy (EELS) method in transmission electron microscopy (TEM) as a quick and straightforward method to determine the indium compositions in (In, Ga)N/GaN NWs with high spatial resolution, independently on the local strain state [5]. In this paper, we will apply this low-loss EELS method to study the lattice pulling effect in ordered (In, Ga)N/GaN NW heterostructures with different indium compositions.

2 Experimental details The axial (In,Ga)N/GaN NW heterostructures studied in this paper were fabricated by selective area growth mode in a plasma-assisted molecular beam epitaxy system equipped with a rf-plasma source for active nitrogen. Firstly, the GaN NWs were grown at $810^{\circ} \mathrm{C}$ under nominally highly metal-rich conditions on GaN-buffered $\mathrm{Si}(111)$ substrates. The GaN buffer was patterned with a $\mathrm{Ti}$ nanohole mask using colloidal 
lithography. The GaN NWs present a good morphological homogeneity containing a pencil-like top [6]. Three (In, $\mathrm{GaN}$ ) NW samples were grown on the top of the nominally identical GaN NWs at the lower temperatures of $550^{\circ} \mathrm{C}$, $500^{\circ} \mathrm{C}$ and $450^{\circ} \mathrm{C}$, respectively, which will systematically increase the $\mathrm{InN}$ mole fraction in the $(\mathrm{In}, \mathrm{Ga}) \mathrm{N}$ section. The other growth parameters were kept constant during the (In, $\mathrm{Ga}) \mathrm{N}$ growth. All the growth details are given elsewhere [6]. Additionally, in situ reflection high-energy electron diffraction was used to monitor the surface morphology and the NW alignment. The optical characterization of the samples was performed using scanning electron microscopy and photoluminescence (PL) spectroscopy.

The (In,Ga)N/GaN NW TEM specimens used for the present study were prepared by standard methods of mechanical grinding and dimpling down to a thickness below $25 \mu \mathrm{m}$. The specimens were then thinned by an argon ion beam with an energy of $3 \mathrm{keV}$ under an incident angle of $3^{\circ}$ at room temperature in a Gatan precision ion polishing system. The Gatan Solarus (model 950) plasma cleaning system is used to remove hydrocarbon contamination on the specimen surface prior to TEM and EELS measurements. The TEM investigation was carried out in a JEOL 3010 microscope with $\mathrm{LaB}_{6}$ cathode operating at $300 \mathrm{kV}$. This microscope is equipped with a post-column Gatan Enfina parallel EELS system that allows for an energy resolution of $1.5 \mathrm{eV}$ according to the full width at half maximum of the zero-loss peak. In the low-loss EELS measurements, typical acquisition times for each spectrum were set to about $0.1 \mathrm{~s}$ using a spot size of about $8 \mathrm{~nm}$ diameter in order to decrease the influence of irradiation damage and hydrocarbon contamination.

3 Results and discussion Figure $1 \mathrm{a}-\mathrm{c}$ presents representative cross-sectional TEM images of $(\mathrm{In}, \mathrm{Ga}) \mathrm{N} / \mathrm{GaN}$ NWs grown on GaN-buffered $\mathrm{Si}$ (111) substrates at $550^{\circ} \mathrm{C}$, $500^{\circ} \mathrm{C}$ and $450^{\circ} \mathrm{C}$, respectively. All NWs are perpendicular to the substrate surface with a total length up to $800 \mathrm{~nm}$. The growth direction of NWs is along the [0001] zone axis, which was determined by convergent beam electron diffraction patterns (not shown here). The strong contrast features visible along the NWs are due to strain-induced bend contours. The NWs contain a low density of threading dislocations and are free of stacking faults. The growth temperature $\left(T_{\mathrm{g}}\right)$ has a strong influence on the NW morphology. On the one hand, the (In, Ga)N NW diameter increases to 200 and $400 \mathrm{~nm}$ for $T_{\mathrm{g}}$ of $500^{\circ} \mathrm{C}$ and $450^{\circ} \mathrm{C}$ due to an enhanced lateral growths (see Fig. $1 \mathrm{~b}$ and c). It is noticed that the lateral growth also occurs in the $\mathrm{GaN}$ segments of the NWs during the (In, Ga)N growth. The reason for that is still under investigation. On the other hand, the tips of the (In, Ga)N NW grown at $500^{\circ} \mathrm{C}$ and $450{ }^{\circ} \mathrm{C}$ exhibit a pencil-like shape, containing inclined $\{1 \overline{1} 02\}$ and $\{11 \overline{2} 2\}$ facets.

The corresponding axial indium distributions at the interface have been investigated by low-loss EELS method [5]. The axial line scans of low-loss EEL spectra
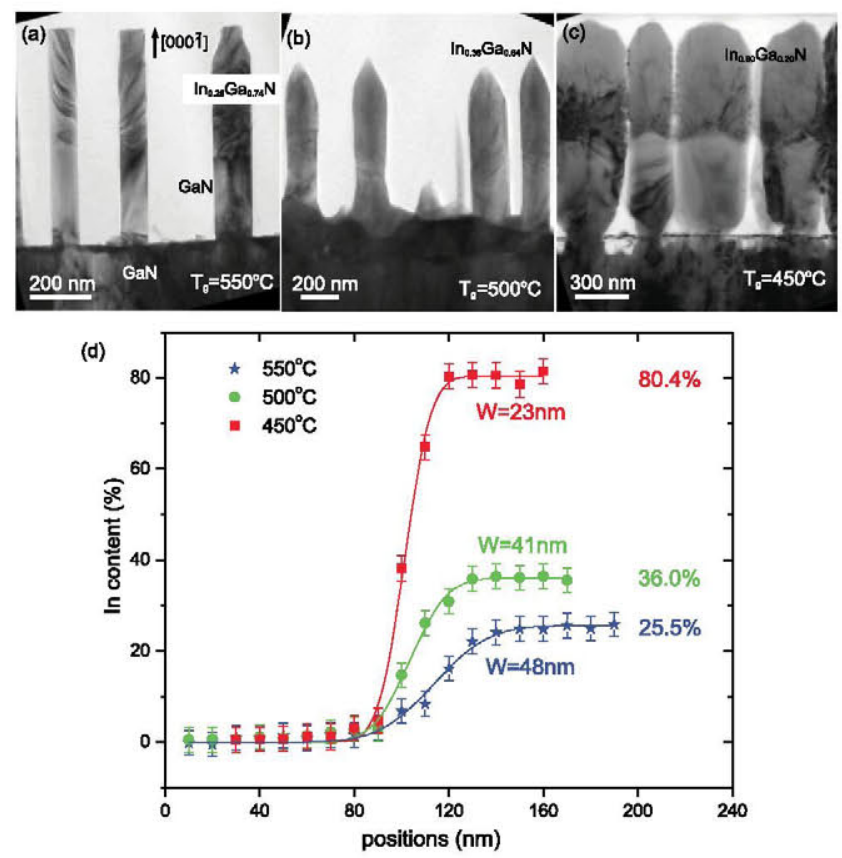

Figure 1 (a), (b) and (c) Cross-sectional TEM images of the axial (In,Ga)N/GaN NW heterostructures grown on GaN-buffered $\mathrm{Si}$ (111) substrate at $550^{\circ} \mathrm{C}, 500^{\circ} \mathrm{C}$ and $450^{\circ} \mathrm{C}$, respectively; and (d) the corresponding indium concentration profiles measured by line scans of low-loss EELS showing the interface widths of 48, 41 and $23 \mathrm{~nm}$ determined by error function fits (solid lines). Error bars are coming from the low-loss EELS measurements [5].

were performed in the center of NWs with a step size of $10 \mathrm{~nm}$ from $\mathrm{GaN}$ to $(\mathrm{In}, \mathrm{Ga}) \mathrm{N}$ sections. The experimental results are shown in Fig. 1d. All three NW heterostructures grown at different $T_{\mathrm{g}}$ exhibit a similar indium composition profile in spite of the distinct indium concentrations. Two main results are notable: (i) The (In,Ga)N NWs have been fabricated with indium concentrations of $25.5,36$ and $80.4 \%$ at $550^{\circ} \mathrm{C}, 500^{\circ} \mathrm{C}$ and $450^{\circ} \mathrm{C}$, respectively, which are in a good agreement to the PL measurements presenting a PL peak shift from $2.3 \mathrm{eV}$ to $1.3 \mathrm{eV}$ [6]. And (ii) all NW samples contain a wide transition region, where the indium content has a gradual evolution and increases along the growth direction of the (In,Ga)N NWs. This result demonstrates the existence of the lattice pulling effect in (In,Ga)N/GaN NW heterostructures, which is consistent with results that we observed before [5]. It is noticeable that the width of the transition region between $(\mathrm{In}, \mathrm{Ga}) \mathrm{N}$ and GaN NWs is remarkably different for the all the samples. In order to quantify this interface width difference, the indium concentration profile is fitted by an error function (cf. solid lines in Fig. 1d). The interface width defined as the distance, where the concentration varies from $10 \%$ to $90 \%$ of the plateau value, is thus determined as 48,41 and $23 \mathrm{~nm}$ for the NWs grown at $550{ }^{\circ} \mathrm{C}, 500^{\circ} \mathrm{C}$ and $450^{\circ} \mathrm{C}$, respectively. The result shows that the interface width becomes smaller and the lattice pulling 

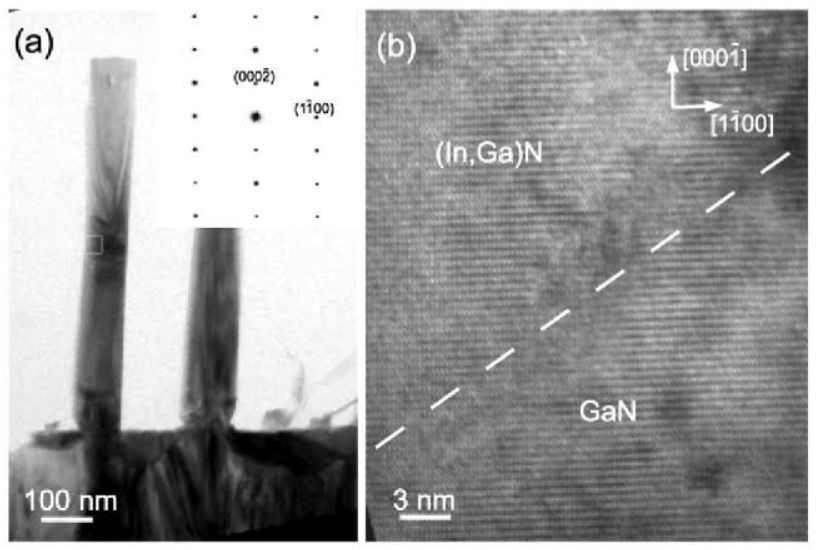

Figure 2 (a) A typical cross-sectional TEM image of axial (In,Ga) $\mathrm{N} / \mathrm{GaN}$ NWs heterostructure grown at $550^{\circ} \mathrm{C}$; inset: the SAED pattern taken in the interface region. (b) Local HRTEM image viewed along $[11 \overline{2} 0]$ zone axis presenting a coherent interface between ( $\mathrm{In}, \mathrm{Ga}) \mathrm{N}$ and $\mathrm{GaN}$ sections.

effect is weakened as the indium concentration increases. This is contradictory to the expectation that $(\mathrm{In}, \mathrm{Ga}) \mathrm{N}$ with a higher indium content has a larger lattice misfit to $\mathrm{GaN}$, and thus, should produce a stronger lattice pulling effect.

In order to clarify the weakening of the lattice pulling effect in NW samples with an increased indium concentration, we carry out the interface analysis in three samples by HRTEM imaging and electron diffraction technique. Figure 2a presents a cross-sectional TEM image of NW sample grown at $550^{\circ} \mathrm{C}$ and a selected area electron diffraction (SAED) pattern taken in the (In,Ga)N/GaN interface region. No splitting of $(1 \overline{1} 00)$ diffraction spot is detected in the SAED pattern. Moreover the local HRTEM image taken along the $[11 \overline{2} 0]$ zone axis (Fig. 1b) reveals a coherent interface between (In, Ga)N and GaN sections. According to the GaN NW tip faceting, the interfaces generally contain $\{1 \overline{1} 02\}$ and $\{11 \overline{2} 2\}$ planes, as marked by the dashed line in Fig. 2b. Both experimental results indicate that, the lattice mismatch in this heterostructure sample is accommodated elastically within the strained (In, Ga)N NWs, while maintaining a coherent interface.

However, when the $T_{\mathrm{g}}$ is lowered to $500^{\circ} \mathrm{C}$ and the indium concentration is increased to $36 \%$, the generation of misfit dislocations is observed at the $(\mathrm{In}, \mathrm{Ga}) \mathrm{N} / \mathrm{GaN}$ interface as shown in Fig. 3. Figure 3c is a (1102) Bragg filtered image originated from the HRTEM image taken in the interface region (Fig. 3b). The filtered image reveals an extra plane in GaN, which is bounded by a misfit dislocation with a Burgers vector component perpendicular to the interface plane normal. The misfit dislocation is pointed out by a white arrow in Fig. 3c. In the sample with a further increase of indium content up to $80 \%$, the corresponding HRTEM image (Fig. 4) shows a high density of misfit dislocations at the interface. The average distance between the misfit dislocations is estimated to be $5 \mathrm{~nm}$.
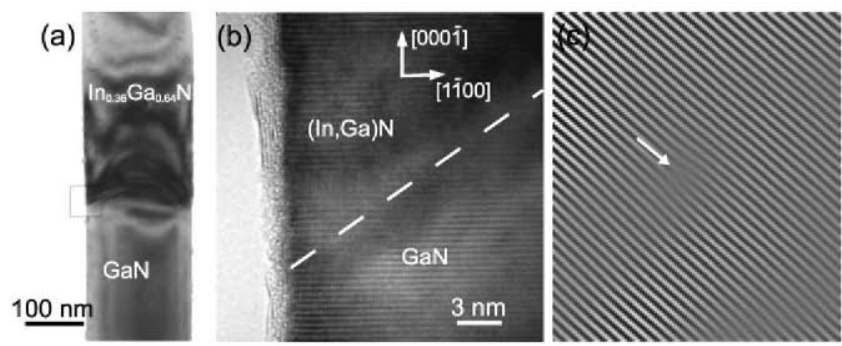

Figure 3 (a) Cross-sectional TEM image of axial (In,Ga)N/GaN NW grown at $500^{\circ} \mathrm{C}$; the interface [11 $\left.\overline{2} 0\right]$ HRTEM image (b) and the corresponding $(1 \overline{1} 02)$ Bragg filtered image (c) revealing the generation of misfit dislocations at the (In,Ga)N/GaN interface (lattice fringe spacing: $0.221 \mathrm{~nm}$ ).
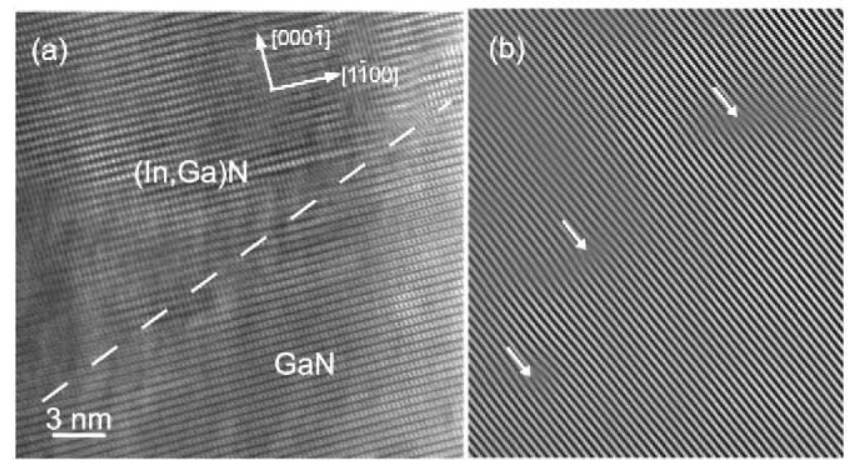

Figure 4 The $[11 \overline{2} 0]$ HRTEM image (a) of the interface in (In,Ga)

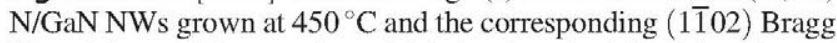
filtered image (b) presenting a high density of misfit dislocations at the $(\mathrm{In}, \mathrm{Ga}) \mathrm{N} / \mathrm{GaN}$ interface (lattice fringe spacing: $0.221 \mathrm{~nm}$ ).

The presence of defects in NW heterostructures grown at lower temperatures demonstrates that the large lattice mismatch due to the higher indium content has to be relieved. The relaxation is achieved not only elastically by the larger free surface at the NW sidewall due to increased NW diameter, but also plastically by the introduction of misfit dislocation at the interface. As the plastic relaxation occurs, the lattice misfit between (In, Ga)N and $\mathrm{GaN}$ is efficiently reduced, and therefore lead to a weaker lattice pulling effects.

4 Conclusions By combing spatially resolved EELS and HRTEM methods, we have demonstrated the impact of the interfacial strain relaxation on the lattice pulling effect and the axial indium composition distributions in the axial (In,Ga)N/GaN NW heterostructures with a indium content up to $80 \%$. The experimental observations show that, as the InN mole fraction increases in (In, Ga)N, the lattice pulling effect is decreased and the interface width is reduced. We attribute the interface width with the degree of the plastic relaxation via the introduction of misfit dislocations at the (In,Ga)N/GaN interface. This result will provide a basic understanding on the indium incorporation in NWs and 
contribute to the design of optimal (In,Ga)N NWs with the desired emission wavelength arrange.

Acknowledgements The authors would like to thank Javier Grandal for critical reading of the manuscript. X. Kong thanks the Key Laboratory of Nanodevices and Applications, Suzhou Institute of Nano-Tech and Nano-Bionics, Chinese Academy of Sciences for the financial support (Grant No 13ZC02).

\section{References}

[1] T. Kuykendall, P. Ulrich, S. Aloni, and P. D. Yang, Nature Mater. 6, 951 (2007).
[2] M. Mandl, X. Wang, T. Schimpke, C. Klper, M. Binder, J. Ledig, A. Waag, X. Kong, A. Trampert, F. Bertram, J. Christen, F. Barbagini, E. Calleja, and M. Strassburg, Phys. Status Solidi RRL 7, 800 (2013).

[3] S. Pereira, M. R. Correia, E. Pereira, K. P. O'Donnell, C. Trager-Cowan, F. Sweeney, and E. Alves, Phys. Rev. B 64, 205311 (2001).

[4] G. B. Stringfellow, J. Cryst. Growth 312, 735 (2010).

[5] X. Kong, S. Albert, A. Bengoechea-Encabo, M. A. Sanchez, E. Calleja, and A. Trampert, Nanotechnology 23, 485701 (2012).

[6] S. Albert, A. Bengoechea-Encabo, M. A. Sanchez-Garca, X. Kong, A. Trampert, and E. Calleja, Nanotechnology 24, 175303 (2013). 